\title{
Intrusion Detection System Employing Multi-level Feed Forward Neural Network along with Firefly Optimization (FMLF2N2)
}

\author{
Komanduri Venkata Sesha Sai Rama Krishna ${ }^{1 *}$, Battula Bhanu Prakash² \\ ${ }^{1}$ Department of CSE, Vignan's Nirula Institute of Technology \& Science for Women, Peda Palakaluru, Guntur 522005, \\ Andhra Pradesh, India \\ ${ }^{2}$ Department of CSE, Tirumala Engineering College, Jonnalagadda, Guntur, India
}

Corresponding Author Email: ksai.mb@gmail.com

https://doi.org/10.18280/isi.240202

Received: 12 January 2019

Accepted: 20 March 2019

\section{Keywords:}

intrusion detection system, KDD info set, firefly Alg, neural network

\begin{abstract}
Number of attacks as of late has immensely expanded because of the expansion in Internet exercises. This security issue has made the Intrusion Detection Systems (IDS) a noteworthy channel for data security. The IDS's are created to in the treatment of attacks in PC frameworks by making a database of the typical and unusual practices for the recognition of deviations from the ordinary amid dynamic interruptions. The issue of classification time is enormously diminished in the IDS through component choice. This paper is proposing the usage of IDS for the successful location of attacks. In view of this, the Firefly Algorithm (FA), another paired element determination calculation was proposed and executed. The FA chooses the ideal number of highlights from NSL dataset. Moreover, the FA was connected with multitargets relying upon the classification precision and the quantity of highlights in the meantime. This is a proficient framework for the discovery of attacks decrease of false alerts. The execution of the IDS in the location of attacks was improved by the proposed classification and highlight choice techniques.
\end{abstract}

\section{INTRODUCTION}

Inferable from basic accessibility moreover broad usage to motorized ambush gadgets, mastermind interferences have transformed into a regular wonder. Today, an a ton of encounters troubles computer structures passing of some sneaky shaped zero-day attempts to computer worms furthermore pollutions arranged for passing on heap stage troubles and also threats [1]. Each last individual about such parts makes system protection an imperative point of view to certain subjective alliance. Some defensive tools like encoding, check, framework association, firewalls and whatnot found to be suggested within the arrangement by means of foremost line of limit across deal with disruptions [2]. Whereas such protective system basically maintains that structure protection, and cannot everything considered square the probability about systems ambushes [3].

Aggressors were ceaselessly discovering theirs path nearby such protective systems for increasing unauthorized approach towards these structures moreover dispatch assaults. Within such manner, rather than such protective steps, Intrusion Detection Systems (IDSs) that flows regarding by means of corresponding next string about structure screen was an earnest part to deterrent on opposition to arrange hazards. An enormous piece about current IDSs use an abuse dependent acknowledgment approach [4], internally that system ambushes were perceived by using an already defined attack marks. In spite of that manner in which the misuse stationed ID method presents a serious shield opposing familiar ambushes, it ignores towards perceive unique and unclear strikes.

Moreover, there is a fundamental interval sneak past within that discovery for next strike furthermore game-plan about its related note [5]. The deferral was routinely exorbitantly clearing moreover been diverse examples for structure assaults, exceptionally zero-day attempts moreover computer worms [6] which has pounded that stamp dependent IDS by means of ludicrous threat for the system [7]. The broad part of the system assaults uses polymorphism which additionally delays the promising era about right engravings. Finally, those made engravings must be confiscated, administrated furthermore sat up with recent through protection executives [8]. Each final one of the variables hold a fundamental hindrance upon the efficiency of outrage dependent IDSs. In this way, certain optional procedure viz. peculiarity dependent intrusion acknowledgment method that is suited for perceiving unique and unclear ambushes a part from previously learning have extended vast certification amongst the examination orchestrate [9].

In this report, we suggest a firefly based IDS utilizing an Artificial Neural Network computation which one is a Multilevel Feed Forward Neural Network (MLF2N2) illustrate [10]. Purpose for working upon ANN dependent IDS show was to exhibit astute master within the structure which is prepared for perceiving these principal models within weird also moreover run of the mill audit records furthermore aggregate up insightful precedent for recent also for subtle survey records. Particular principal favored point of view for neural framework dependent IDS exhibit its strength to address the complicated non-coordinate speculations which can't be addressed with a straight classifier technique as Naive Bayes [11]. The designed MLF2N2 dependent IDS demonstrate utilizes firefly upgrade methods to restrict those all things considered estimation over drew within the readiness and also implementation. Execution examination of designed model upon standard NSL-KDD dataset [12] displays that it plays out 
all around stood out from other interference distinguishing proof models reliant on SVM, Naive Bayes and C4.5 counts [13].

The straggling residue of this report has been sorted out in below ways. Portion II accord related tasks. Zone III formats numerical review for neural framework moreover a general delineation of in what way it can be used to improve an interruption identification show. Zone IV depicts the proposed interruption discovery show. Section V presents test outcomes. End and future work were discussed in Section VI.

\section{LITERATURE WORK}

As of late fluctuated methods that were anticipated also created for interruption recognition framework; Intrusion Detection Systems can be a component which shields assets moreover learning from unapproved authorities, abuse, furthermore pernicious interruptions within an extremely dispersed registering air [14]. the associated methodology for interruption recognition framework ar like fake neural system (ANN), Rule based generally order, gathering, various agglomeration calculations like $\mathrm{K}$-implies agglomeration, $\mathrm{K}$ implies agglomeration with fluffy algorithmic program, Radial premise work (RBF) and Self sorted out guide organize (SOM), Support Vector Machines, Naïve Bayesian Classifiers, and so on this allows the model to discover unusual/abnormal conduct and respond with the worthy reaction [15].

At any rate starting late the head of the arithmetic plan also has been foreseen furthermore made of the interference ID system to defeat suitable reaction of earlier approach inside the area of learning accumulation moreover course of action to improve the speed for disclosure, As Support vector machine as a portion upon the likelihood for collection, which portrayed the data within a to a great degree higher route than that of alternative existing methods [16]. Machine learning frameworks likewise utilized for orchestrated the data inside the conditions of oversaw, half coordinated and unsubstantiated methodologies within misuse a couple of data planning approach. Starting at now the head of the algorithmic program works with the informational index in an amazingly prevalent path along hymenopter area headway (ACO), Particle of swarm streamlining (PSO), inherited algorithmic steps furthermore two or three elective upgrade counts for highest distinguishing proof either enhance the speed for area of the interference acknowledgment structure [17].

A. Chnadrasekhar as well as K. Raghuveer et al. [18] a fresh out of the box new methodology for information preparing procedures like neural fluffy with exploitation bolster vector machine underpins into enhance the identification rate of partner degree interruption recognition framework, entire work is part into four totally unique advances beginning produce the sub set with the help of $\mathrm{k}$ implies that agglomeration at that point prepared all the sub set with exploitation neuro fluffy manage show at that point grouped the successive learning with the help of SVM and in the long run discover with the RBF neural system [19].

Shen Li, Feng statue maker et al. [3] at this time they foreseen accomplice degree intrusion area setup known as NTDERC reinforced obnoxious sets information dealing with framework. The thought is to utilize the distinctive cruel classifiers moreover the upsetting meta-learning plan tactic as instruments to study arrange development and moreover to improve interference area structures. Examinations within
1999 DARPA learning set shows gathering brutal classifiers technique has higher DR rate and moreover the less metal rate than unique unpalatable classifier theory. Machine learning methods is an important space to intrusion acknowledgment, these regular courses to accomplice degree associate degree omaly interference area structure was asserted to coordinated the readiness procedure for data game plan in an amazingly greater way moreover to improve the speed of revelation.

Feng Guorui, Zou Xinguo as well as Shanghai vernacular Jian et al. [5] foreseen a semi-managed learning algorithm for intrusion acknowledgment is that those foreseen courses joined within the Fuzzy C-Means algorithmic code. The affectability for starting characteristics measures moreover the shot for lodgings within neighborhood perfect are fantastically diminished through abuse many checked figuring out how to update the training capacity of the fleecy agglomeration suggests that algorithmic code. The KDDCUP ninety nine data firm is grasped in light of the way that the preliminary subject at the information cost which contains standard and moreover peculiar cost of dataset.

Mohammed Hasan Ali et.al proposed "another interference revelation structure subject to Speed Learning Network and also Particle swarm upgrade" Supervised Intrusion Detection System a system which has the capacity to pick up from models regarding past assaults to recognize recent ambushes. Utilizing ANN based intrusion revelation is hopeful for decreasing the rate of false negative or also false encouraging points in light of the way that ANN has the limit of picking up from genuine models. This article defines made learning model for Fast Learning Network (FLN) in light of particle swarm headway (PSO) which has been proposed and also called as PSO-FLN. The pattern might have been associated with issue about intrusion disclosure also endorsed reliant on acclaimed dataset KDD99.

\section{PROPOSED WORK}

This section explains proposed model of multi-level network and also firefly model. Further elucidate about system model and its works.

\subsection{Multilayered neural network}

A multi-layer neural system contains in excess of one layer of fake neurons or hubs. They contrast generally in outline. It is vital to take note of that while single-layer neural systems were helpful right off the bat in the advancement of AI, by far most of systems utilized today have a multi-layer display [20]. Multi-layer neural systems can be set up from multiple points of view. Normally, they have something like one info layer, which sends weighted contributions to a progression of concealed layers, and a yield layer toward the end [21]. These more advanced setups are additionally connected with nonlinear forms utilizing sigmoids and different capacities to coordinate the terminating or initiation of fake neurons. While a portion of these frameworks might be constructed physically, with physical materials, most are made with programming capacities that model neural movement.

Let $x=$ No. of info somatic cell

$y=$ No. of somatic cell in the shrouded layer.

$z=$ No. of yield somatic cell. 
$K_{p q}=$ Weight interfacing with the somatic cell in the info layer to the $q^{\text {th }}$ somatic cell in the concealed layer $p=$ $1,2, \ldots, x$ and $q=1,2, \ldots, y$.

$S_{n o}=$ Weight associating with the $\mathrm{n}$ somatic cell within concealed level to the o somatic cell within yield layer $q=$ $1,2 \ldots \ldots, y, o=1,2, \ldots, k$. The NET an incentive for $\mathrm{q}$ somatic cell in the concealed layer shown as:

$$
A_{q}=\sum_{p=1}^{x} p_{d} d_{p q}
$$

Employing the exchange work, yield an incentive at the shrouded layer is shown as:

$$
E_{q}=G\left(A_{q}\right)=\frac{1}{\left(1+\exp \left(-A_{q}\right)\right)}
$$

The NET an incentive for $c^{t h}$ neuron within the yield layer is as given as:

$$
N E T_{c}=\sum_{q=1}^{y} E_{q} S_{n o}
$$

Applying the enactment work,

$$
O U T_{o}=G\left(N E T_{o}\right)=\frac{1}{\left(1+\exp \left(-N E T_{o}\right)\right)}
$$

Applying the activation function,

\subsection{The firefly algorithm}

The main role for a firefly's blaze is to go about as a flag framework to draw in different fireflies. Xin-She Yang figured this firefly calculation by assuming:

(1). All fireflies are unisexual, so any individual firefly will be pulled in to every single other firefly;

(2). Attractiveness is relative to their splendor, and for any two fireflies, the less splendid one will be pulled in by (and subsequently move towards) the more splendid one;

(3). In any case, the force (obvious brilliance) diminish as their shared separation increments; if there are no fireflies more splendid than a given firefly, it will move randomly. The brilliance ought to be related with the goal work.

After induction, each firefly is considered in contradiction of completely the rest, and determination move towards each more awe inspiring firefly qualified. At the point when a firefly has chosen it needs to change near an all the more scandalous firefly, some effects need to happen. At first, the departure among the fireflies, w, must be assumed. Any sort of departure calculation that examines valuable for the given issue can be exploited, so far for the over-all instance the Cartesian division is correct. The Cartesian departure among two fireflies in R-dimensional space is assumed as:

$$
Q_{u v}=\sqrt{\sum_{r=1}^{R}\left(c_{u r}-c_{v r}\right)^{2}}
$$

where $o_{u}$ and $o_{v}$ are the location vectors for firefly $\mathrm{u}$ and $\mathrm{v}$ correspondingly with $o_{u}(1)$ representing the position value for the $1^{\text {th }}$ dimension [22].

With the departure of two fireflies identified, the engaging quality q can be resolved. Meanwhile the appeal depends on the light force, double terms go into the figuring of $q_{1}$. In the major place, from the backwards four-sided law $q_{1}(k)=$ $i_{0} / k^{2}$, wherever the power of the firefly presence stimulated near[23]. At that point because of the retention through the air, $q_{2}(k)=i_{0} / e^{-w k}, \mathrm{w}$ is the assimilation constant, and $w €[0, \infty)$. $\mathrm{w}$ of 0 vintages no retention, although a vast w identifies with the fireflies flying within a substantial haze. At that point to create an estimate of the consolidated terms, and to stay away from the unclear consequence $q_{1}(0)$, we get $q(k)=i_{0} / e^{-w k^{2}}$. In any case, computing an exponential is costly, and $i_{0} / e^{-w k^{2}}$ can be approached by $i_{0} /\left(1+w k^{2}\right)$, which isn't as hard to figure. Since the allure is specifically identified with the light force $q_{0}=i_{0}$, where $q_{0}$ is the engaging quality at $\mathrm{k}=0$, ordinarily $q_{0} €(0,1]$. Consolidating the least two comments outputs the articulation $q(k)=q_{0} /\left(1+w k^{2}\right)$. Changing $q_{0}$ changes how pulled in fireflies are to other people, so bringing down $\mathrm{q}_{0}$ brings down the craving for a firefly to move towards more brilliant fireflies.

$$
o_{i}=o_{i}+q_{0} /\left(1+w k_{x y}^{2}\right)\left(o_{v}-\mathrm{u}\right)+p A(B-0.5)
$$

Here, B is a strategy of dependably taken emotional statistics within the degree of $[0,1]$, and it is a limit regulatory to extent for haphazardness. Tact parameter $\mathrm{p}$ wasreliably within the range $[0,1]$ here 0 relates as no oddity also 1 takes a gander at presence very optional.

\section{The firefly algorithm}

Given The:

Target work $\mathrm{f}(\mathrm{o})$, in which $\mathrm{o}=\left(\begin{array}{llll}\mathrm{o}_{1}, \mathrm{o}_{2} & \ldots & \mathrm{o}_{\mathrm{r}}\end{array}\right)$ for $\mathrm{r}$ measurements.

Light power Ii upon oi was dictated through f(oi)

Characterize $\mathrm{p} \mathrm{q} \mathrm{w}$ and $\mathrm{t}$

Given arbitrary areas for $\mathrm{N}$ fireflies within y measurements

Beginning assessment for total $\mathrm{N}$ fireflies

While (End condition not encountered)

Summation $\mathrm{t}$

For $\mathrm{u}=1$ to Num Fireflies

For $\mathrm{v}=1$ to Num Fireflies

In the event that $\left(I_{v}>I_{u}\right)$

Move firefly $\mathrm{u}$ towards $\mathrm{v}$ in $\mathrm{r}$-measurement

End if

End for $\mathrm{n}$

Assess novel arrangement also refresh light force

Check until this point that the best position found or not

End for $\mathrm{u}$

Decrease the alpha

Sort fireflies

End

\subsection{System paradigm}

Here we suggest our IDS and also next shows how our arrangement make sense of the issues declared earlier.

Proposed IDS was sorted as feed-forward neural associated along back-spread figuring. Neural framework characteristics like parallelism, passed on count, learning capacity, adaptively furthermore adjustment to un-basic disappointment makes 
sensible of intrusion recognizable proof structures [24]. Moreover as feed-forward neural framework can evaluate every limit with needed precision [12] furthermore its openness across various next neural frameworks, we choose this framework for our IDS. Here we introduce the framework display of IDS framework dependent on Multi-layer feed forward neural system using firefly advancement [25]. The picture demonstrates to work proposed strategy. This method consists three parts first one is initial part here first we have to take data set that is KDD data set for our proposed method. Now we would preprocess the data set after preprocessing we just proceed to train of MLF2N2.second part is training part here we will choose set of initial inputs for model next that it will refresh. Generally this model takes huge time, so that we applying firefly instead of training. The proposed method reduces the execution time.

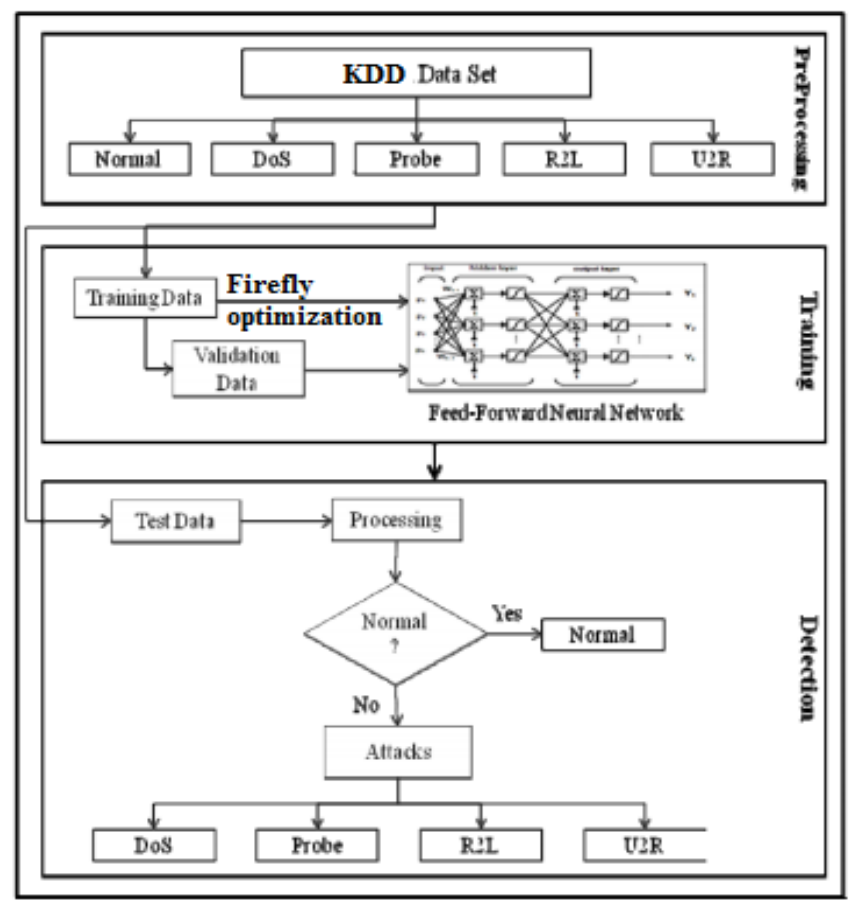

Figure 1. System paradigm

\subsubsection{Preliminaries}

Define occupants of fireflies, $\mathbf{C}_{\mathbf{s}, \mathbf{t}}$

Where $\mathrm{s}=1,2,3, \ldots \mathrm{n}$

$\mathrm{t}$ stands the choice inconstant number

What's more, $\mathrm{n}$ is the inhabitant's measure

Particular decision factors designed to the firefly were the quantity for hubs within shrouded film for MLFNN, $\mathrm{C}_{\mathrm{i}, 1}$

Erudition rate, $\mathrm{C}_{\mathrm{i}, 2}$,

Also the impetus rate, $\mathrm{C}_{\mathrm{i}, 3}$,

Here target work was to limit RMSE. RMSE of MLFNN, was figured utilizing

$$
\operatorname{RMSE}=\sqrt{\sum_{\mathrm{d}=1}^{\mathrm{n}}\left(\mathrm{Z}_{\mathrm{e}, \mathrm{d}}-\mathrm{Z}_{\mathrm{f}, \mathrm{d}}\right) \wedge 2 \div \mathrm{m}}
$$

Here $\mathrm{n}$ is the quantity for information designs. $\mathrm{Z}_{\mathrm{e}, \mathrm{d}}$ and $\mathrm{Z}_{\mathrm{f}, \mathrm{d}}$ were anticipated furthermore objective estimation for the yield individually.

$$
\mathrm{C}^{\prime} \mathrm{s}, \mathrm{t}=\mathrm{Cs}, \mathrm{t}+\beta \mathrm{iq}(\mathrm{Cs}, \mathrm{t}-\mathrm{C} \mathrm{k}, \mathrm{t})+\alpha(\text { rand }-1 / 2)
$$

wherever $\alpha$ the random constraint and rand is an irregular number somewhere in the range of 0 and 1 . The engaging quality of firefly 'an' as for firefly 'b', is $\beta i k$ figured using

$$
\beta_{i k}=\beta_{0} e^{-\gamma u i k 2}
$$

wherever $\beta 0$ is the engaging quality of firefly as soon as $u=0$, $\gamma$ known as the light retention factor for media.

Now, departure among firefly ' $s$ ' and ' $t$ ', $\gamma_{\text {st }}$ was resolved utilizing

$$
\gamma_{\mathrm{st}}=\sqrt{\sum \underbrace{\mathrm{e}}_{\substack{\mathrm{s}=1 \\ \mathrm{~s}=1}}-\mathrm{C}_{\mathrm{k}, \mathrm{t}})^{2}}
$$

\subsubsection{Algorithm for FMLF2N2 Begin}

\section{\{}

a)Caluclate RMSE Value of MLFLN using equation 1

b) Assess the power of light, Ia for every firefly.

Ia, was running within wellbeing regard gotten through figuring the RMSE within the wake of simulating that MLFNN by means about relating estimations of $\mathrm{Ca}, 1, \mathrm{Ca}, 2$, and $\mathrm{Ca}, 3$ for every one firefly.

During this step, getting ready data is rummage-sale of recreating MLFNN.

c) Designed for a firefly an in the m people, In case (RMSE (h) greater to RMSE (l))

\{

Change firefly ' $h$ ' close firefly 'l' using equation 2 \}

d) Resulting to reviving these circumstances of firefly ' $h$ ' by figuring new estimation of $\mathrm{C}(\mathrm{h}, \mathrm{b})$ for all estimations of $\mathrm{k}$

Using equation 2, specific light concentration of light for firefly ' $h m$ ' is invigorated by enrolling the RMSE.

Fresh course to action of choice elements moreover RMSE were set as the revived characteristics for firefly ' $h$ '.

e) Recurrence organize 4 until total fireflies within the people were differentiated and firefly ' $h$ '. All through every evaluation, the contrasting firefly was set to firefly 'l'.

f) Set up the varying firefly within ' $n$ ' tenants to be firefly 'h'.

Accentuation arrange 4 also 5 till the point that the moment that all fireflies are composed among themselves.

Around the complete of this stage, another social occasion of ' $m$ ' fireflies is encircled and record as the general population for the following get-together.

g) Abundant the new assembling of ' $m$ ' fireflies in climbing demand giving to the looking at RMSE.

Thusly, the current present better firefly will be orchestrated at most elevated purpose for overgrown.

h) Recurrence organize 4,5 and 6 until the point that the moment that a most extraordinary number of age is expert.

The finest firefly of an age which makes the most insignificant RMSE is doled out as the overall finest firefly or the perfect game plan.

i) Except the estimation the count of center points for covered part,

Erudition rate as well as power rate got from overall best firefly as parameters of the MLFNN.

j) Behavior particular testing strategy through reproducing saved MLFNN within the testing data 
Furthermore, deal within the RMSE of model.

End

\}

\section{EXPERIMENTAL LAYOUT}

Here, we are using python 3.6 on Anaconda navigator by using spyder [26]. The trials were performed on an Intel Core 3 Duo Processor and 4 GB RAM and 1TB HDD is used to running on the stage Microsoft Windows 8.

\subsection{Results and discussions}

Here, we plan also test our structure with KDD datasets within contrasting count of relationship within the set. Within every get-together, $80 \%$ of the information was used for plan remaining $20 \%$ for information of test [27]. To get ready and organize, we separate the few counts of underwriting datasets. The framework was kept on running under each support set and final outcomes were enrolled for every get-together. Among all chooses, we close confirmation for $20 \%$ of preparing information like support set, is perfect decision also results in the good acknowledgment rates.

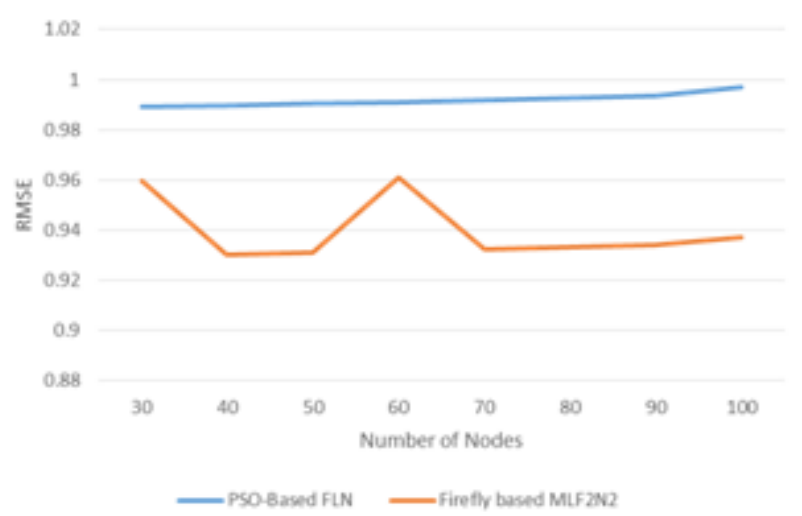

Figure 2. RMSE value comparison

Figure 2 demonstrates the comparasion for being strategy and proposed technique for mistake rate. Proposed strategy blunder rate is diminish in staggered system moreover firefly combiation make preparing all the most effectively.

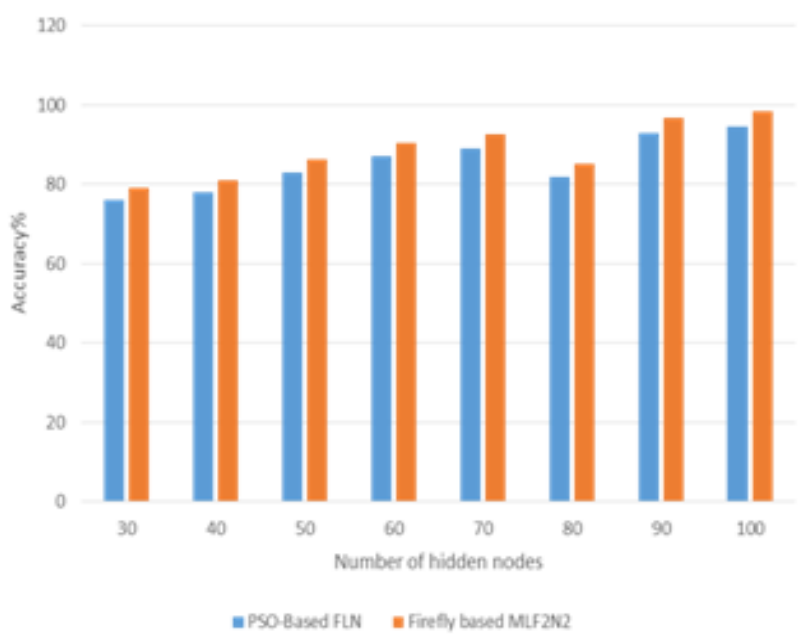

Figure 3. Accuracy of proposed and existing
Figure 3 demonstrates about comparasion for being work exactness also proposed work, because of hence blunder rate is high in staggered arrange for inverse end PSO-dependent FLN has low precision due to slip-up rate also preparing period and learning period. Addition for covered neuron's count associated with the extension of precision. Our's examination within perplexity orchestrate outcomes can be imparted by means of looks for after. R2L strike, have got cut down accuracy separating and differing areas. It is required to that obliged extent for preparing data within the class separating and also various classes. In any situatuin, there is an unmistakable case for pushing ahead exactness within augmentation for measure to neurons within concealed layer.

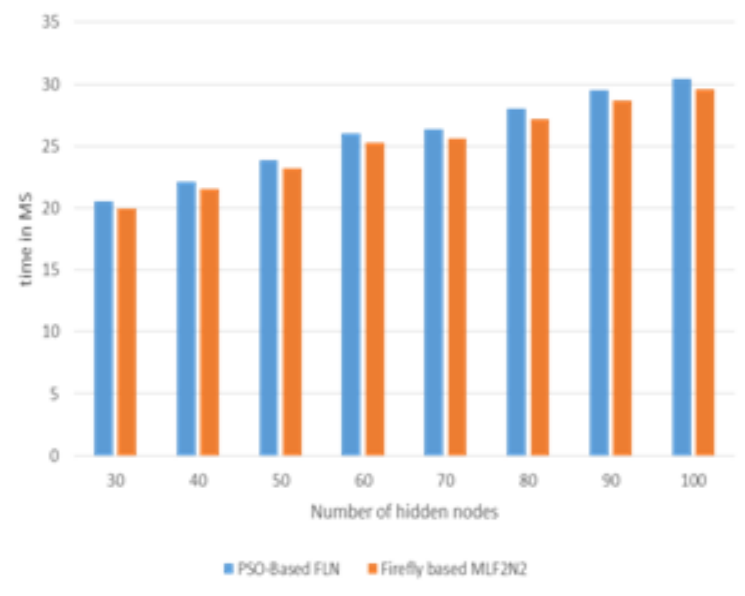

Figure 4. Training time

Here, we get ready also trail our structure within KDD datasets also along different count of affiliations. Within every social affair, $80 \%$ data was utilized as getting ready data furthermore $20 \%$ as the test data. Within planning stage, we take a gander at a couple of count of endorsement datasets. Then the system is continued running within every endorsement set furthermore last outcomes were enlisted for every get-together. Amongst these conclusions, here we complete decision of $20 \%$ for getting ready data as an endorsement set, which was the best choice moreover results within the better disclosure rates. Here figure 4 exhibits planning period connection among existing and also proposed strategies with moving the amount of hidden center points within the framework. When amount of getting ready centers increases time will be diminished.

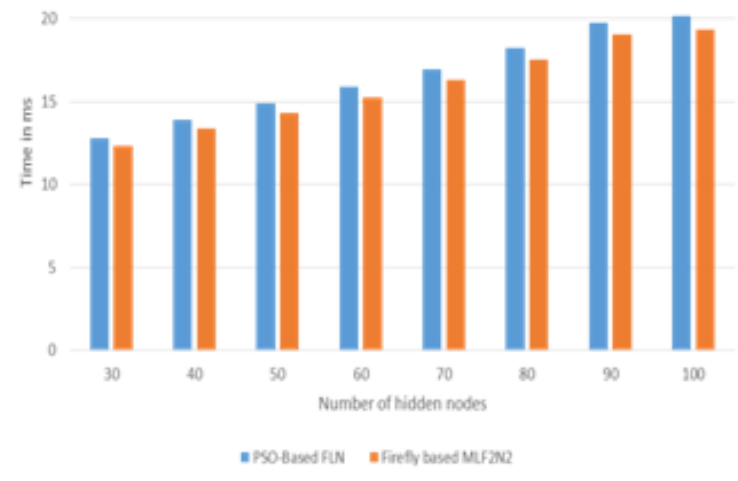

Figure 5. Testing time 
Figure 5 demonstrate testing period examination among the existing and also proposed techniques by moving amount of disguised center points within the framework. Whenever we augment that amount for centers testing period will diminished.

In the wake of getting ready also testing for the F-MLF2N2, execution for F-MLF2N2 was differentiated. The outcomes were portrayed in Figures 4, 5, 6 and 7. FA-MLFNN has beaten the PSO-FLN by making low RMSE in the midst of both getting ready for testing shapes. The FA-MLFNN has ceded RMSE which was approximately $3.980 \%$ and $27.34 \%$ lesser than RMSE occurred by PSO-FLN in the midst of getting ready moreover testing independently.

\section{CONCLUSION}

Here in the paper, the problem about intrusion recognizable proof has revealed moreover within writing we inspected that approaches of fathoming. The present techniques were nope up to the stamp since they ought to be done quantifiably subject to couple of properties. With the true objective to handle that we displayed a method that relies upon ANN which was amazed feed forward neural framework within a redesigning part for firefly known as FMLF2N2. Utilizing FMLF2N2 dependent intrusion acknowledgment is all the more consoling for diminishing the amount of mistaken negative or false positives in light of the fact that FMLF2N2 has the limit of picking up from genuine models. The model has been associated with the issue of interference disclosure and affirmed reliant upon praised dataset KDD99. The proposed method has taken a gander at against PSO-FLN strategy. It might be thought that the made FMLF2N2 defeated the another learning approaches within the testing exactness of learning.

\section{REFERENCES}

[1] Solangi, K.H., Islam, M.R., Saidur, R., Rahim, N.A., Fayaz, H. (2011). A review on global solar energy policy. Renewable and Sustainable Energy Review, 15: 21492163. https://doi.org/10.1109/ACCT.2012.15

[2] Zhang, P., Li, W., Li, S., Wang, Y., Xiao, W. (2013). Reliability assessment of photovoltaic power systems: Review on current status and future perspectives. Applied Energy, 104: 822-833. https://doi.org/10.1016/j.apenergy.2012.12.010

[3] Mellit, A., Pavan, A.M., Kalogirou, S.A. (2011). Application of artificial neural networks for the prediction of a $20-\mathrm{kWp}$ grid-connected photovoltaic plant power output. Soft Computing in Green and Renewable Energy Systems, 269: 261-283. https://doi.org/10.1007/978-3-642-22176-7_10

[4] Zainuddin, H., Shaari, S., Omar, A.M., Sulaiman, S.I. (2011). Operating temperature correlation with ambient factors of Building Integrated Photovoltaic (BIPV) GridConnected (GC) system in Malaysia. International Review on Modelling and Simulations, 4.

[5] Sulaiman, S.I., Rahman, T.K.A., Musirin, I. (2009). Prediction of grid photovoltaic system output using evolutionary programming-ANN models. in proc. 8th WSEAS International Conference on Artificial Intelligence, Knowledge Engineering, \& Databases, pp. 176-180. https://doi.org/10.1080/15502280903106465
[6] Kanat, Z.E., Özdil, N. (2013). Application of artificial neural network (ANN) for the prediction of size of silver nanoparticles prepared by green method. The Journal of The Textile Institute, 109(9): 1247-1253. https://doi.org/10.1080/00405000.2017.1423003

[7] Meireles, M.R.G., Almeida, P.E.M., Simoes, M.G. (2003). A comprehend sivereview for industrial applicability of artificial neural networks. IEEE Trans. Industrial Electronics, 50: 585-601.

[8] Yang, X.S., He, X. (2013). Firefly algorithm: Recent advances and applications. Int. J. Swarm Intelligence, 1: 36-50. https://doi.org/10.1504/IJSI.2013.055801

[9] Pal, S.K., Rai, C.S, Singh, A.P. (2012). Comparative study of firefly algorithm and particle swarm optimization for noisy non-linear optimization problems. Int. J. on Intelligent Systems and Applications, 10: 50-57. https://doi.org/10.5815/ijisa.2012.10.06

[10] Sulaiman, S.I., Rahman, T.K.A., Musirin, I., Shaari, S. (2011). Evolutionary programming versus artificial immune system in evolving neural network for gridconnected photovoltaic system output prediction. WSEAS Transactions on Systems and Control, 6: 197206.

[11] Huang, G.B., Zhu, Q.Y., Siew, C.K. (2006). Extreme learning machine: Theory and applications. Neurocomputing, $\quad$ 70(1-3): 489-501. https://doi.org/10.1016/j.neucom.2005.12.126

[12] Huang, G.B., Zhu, Q.Y., Siew, C.K. (2004). Extreme learning machine: A new learning scheme of feedforward neural networks. Neural Networks, 2004. Proceedings. 2004 IEEE Int. Jt. Conf., 2(8): 985-990. https://doi.org/10.1109/IJCNN.2004.1380068

[13] Li, G., Niu, P., Duan, X., Zhang, X. (2014). Fast learning network: A novel artificial neural network with a fast learning speed. Neural Comput. Appl, 24(7-8): 16831695. https://doi.org/10.1007/s00521-013-1398-7

[14] Wang, J., Wu, W., Li, Z., Li, L. (2011). Convergence of gradient method for double parallel feedforward neural network. Int. J. Numer. Anal. Model, 8(3): 484-495.

[15] Sahu, S.K., Sarangi, S., Jena, S.K. (2014). A detail analysis on intrusion detection datasets. Souvenir 2014 IEEE Int. Adv. Comput. Conf. IACC 2014, 1348-1353. https://doi.org/10.1109/IAdCC.2014.6779523

[16] Olusola, A.A., Oladele, A.S., Abosede, D.O. (2016). Analysis of KDD' 99 intrusion detection dataset for selection of relevance features. Lecture Notes in Engineering and Computer Science, 2010(1): 16-23. https://doi.org/10.1117/12.929089

[17] Chou, T.S., Fan, J., Fan, S., Makki, K. (2009). Ensemble of machine learning algorithms for intrusion detection. 2009 IEEE Int. Conf. Syst. Man Cybern, pp. 3976-3980. https://doi.org/ 10.1109/ICSMC.2009.5346669

[18] Trelea, I.C. (2003). The particle swarm optimization algorithm: Convergence analysis and parameter selection. Inf. Process. Lett, 85(6): 317-325. https://doi.org/10.1016/S0020-0190(02)00447-7

[19] Blondin, J. (2009). Particle swarm optimization: A tutorial. $\quad .$. Site Http//Cs. Armstrong. Edu/Saad/Csci8100/Pso Tutor. 1-5.

[20] Sengupta, A., Bhadauria, S., Mohanty, S.P. (2017). TLHLS: Methodology for low cost hardware trojan security aware scheduling with optimal loop unrolling factor during high level synthesis. IEEE Trans. Comput. Des. Integr. Circuits Syst, 36(4): 660-673. 
https://doi.org/10.1109/TCAD.2016.2597232

[21] Mishra, V.K., Sengupta, A. (2015). Swarm-inspired exploration of architecture and unrolling factors for nested-loop-based application in architectural synthesis. Electron. Lett, 51(2): 157-159. https://doi.org/10.1049/el.2014.3507

[22] Sengupta, A., Bhadauria, S. (2015). User power-delay budget driven PSO based design space exploration of optimal k-cycle transient fault secured data path during high level synthesis. Sixteenth International Symposium on Quality Electronic Design, 2(6): 289-292. https://doi.org/10.1109/ISQED.2015.7085441

[23] Mishra, V.K., Sengupta, A., (2014). MO-PSE: Adaptive multi-objective particle swarm optimization based design space exploration in architectural synthesis for application specific processor design. Advances in Engineering Software, 67: 111-124. https://doi.org/10.1016/j.advengsoft.2013.09.001

[24] Sengupta, A., Mishra, V.K. (2014). Expert Systems with Applications Automated exploration of data path and unrolling factor during power - performance tradeoff in architectural synthesis using multi-dimensional PSO algorithm. Expert Systems with Applications, 41(10): 4691-4703. https://doi.org/10.1016/j.eswa.2014.01.041

[25] Shi, Y., Eberhart, R. (1998). A modified particle swarm optimizer. 1998 IEEE International Conference on Evolutionary Computation Proceedings. IEEE World Congress on Computational Intelligence (Cat. No.98TH8360), pp. 69-73. https://doi.org/10.1109/ICEC.1998.699146

[26] Li, G., Niu, P., Zhang, W., Liu, Y. (2013). Model NOx emissions by least squares support vector machine with tuning based on ameliorated teaching-learning-based optimization. Chemometrics and Intelligent Laboratory Systems, 126:

11-20. https://doi.org/10.1016/j.chemolab.2013.04.012

[27] Tvrdik, J. (2006). Competitive differential evolution and genetic algorithm in GA-DS toolbox. Tech. Comput. Prague, Praha, Humusoft, (1): 99-106. 